\title{
La secularización en el contexto sociocultural latinoamericano
}

\section{Luis Vicente Sepúlveda Romero*}

Recepción: 15 de agosto de 2018 • Aprobación: 12 de septiembre de 2018

\section{Resumen}

La propuesta de este artículo plantea investigar la comprensión de la secularización como nuevo escenario cultural en el que transitan los discursos religiosos. En este sentido, se indaga, en la conmemoración de los cincuenta años del Documento de Medellín, una aproximación al concepto de secularización, que recoge las distintas formas de entender lo religioso en la posmodernidad. De ahí que estas nuevas comprensiones puedan ayudar a contextualizar la reflexión teológica contemporánea en un diálogo con las ciencias sociales.

Palabras clave: ciencias sociales, Iglesia latinoamericana, secularización, signos de los tiempos, teología.

Artículo de reflexión preparado para el Primer Congreso Internacional de Teología Latinoamericana y del Caribe: 50 años de Medellín: Iglesia y signos de los tiempos, desarrollado en la Universidad Santo Tomás entre el 16 y el 19 de octubre de 2018. Citar como: Sepúlveda Romero, L. V. (2018). La secularización en el contexto sociocultural latinoamericano. Albertus Magnus, IX(2), 107-118. Doi: https://doi.org/10.153322/5005413.5103.

* Colegio Nacionalizado La Presentación, Colombia. Correo electrónico: vicente. sepulveda@gmail.com 


\title{
Secularization in the Latin American sociocultural context
}

\begin{abstract}
The proposal of the paper raises questions about the understanding of secularization as a new cultural scenario in which religious discourses reside. In this sense, the framework of the commemoration of the 50th anniversary of the Document of Medellín is explored, an approach to the concept of secularization, which includes the different ways of understanding the religious in postmodernity. Hence, these new understandings can help to contextualize contemporary theological reflection in a dialogue with the social sciences.

Keywords: social sciences, Latin American Church, secularization, signs of the times, theology.
\end{abstract}

\section{A secularização no contexto sociocultural latino-americano}

\section{Resumo}

A proposta deste artigo expõe pesquisar a compreensão da secularização como novo cenário cultural no qual transitam os discursos religiosos. Neste sentido, indaga-se na comemoração dos cinquenta anos do Documento de Medellín, uma aproximação ao conceito se secularização, que coleta distintas formas de entender o religioso na pós-modernidade. Daí que estas novas compreensões possam ajudar a contextualizar a reflexão teológica contemporânea em um dialogo com as ciências sociais.

Palavras-chave: ciências sociais, Igreja latino-americana, secularização, signos dos tempos, teologia. 


\section{Introducción}

La ruta que se propone para esta reflexión parte, en primer lugar, de una mirada global a los contextos de América Latina hacia finales de la década de 1960 a partir del acontecimiento de Medellín. Esto nos ubica en dos horizontes: el primero da cuenta de la complejidad de una lectura en la que confluyen elementos culturales, económicos, políticos y, por supuesto, eclesiales de todo un continente, lo que implica que solo en algunos asuntos es posible la generalización. La II Conferencia General del Episcopado Latinoamericano realizó la recepción del Vaticano II que, en el espíritu de la reforma conciliar, intenta dilucidar el papel de la Iglesia latinoamericana en un contexto sociocultural secularizado. En segundo lugar, se explora la categoría secularización como posibilidad hermenéutica para la teología con el propósito de dar cuenta de las transformaciones del mapa religioso latinoamericano.

\section{Contexto sociocultural latinoamericano}

Pensar en la II Conferencia General del Episcopado Latinoamericano, reunida en Medellín del 26 de agosto al 8 de septiembre de 1968, remite al acontecimiento eclesial más importante del siglo XX: el Concilio Vaticano II. Este evidencia el querer de la Iglesia de pasar, de un modelo caracterizado por una institución jerárquica vista como societas perfecta, a una Iglesia que, por el contrario, se fortalece en el magisterio pontificio y episcopal "que es de comunión y participación, encarnada en las realidades sociales; que cree en la propiedad al servicio del bien común; que separa a la Iglesia de los poderes dominantes, y que se compromete con las mayorías empobrecidas" (Sactena, 2016, p. 655).

El papa Juan XXIII, al convocar el Concilio, daba inicio al proceso de aggiornamento, entendido como puesta al día de la Iglesia frente a los retos y los cambios del mundo contemporáneo. Allí radica la importancia del acontecimiento de Medellín, ya que le correspondió dinamizar este proceso de puesta al día eclesial en el contexto latinoamericano y generar en sí misma una identidad eclesial latinoamericana (Luciani, 2018).

Al realizar una aproximación a la realidad cultural de la región, se podría afirmar, junto con Rodrigo Guerra, que "podemos tener al menos la certeza de que nuestra cultura de base es al menos la versión no-ilustrada de la modernidad que se encuentra fuertemente marcada por el acontecimiento cristiano" (Consejo Episcopal Latinoamericano [Celam], 2005, p. 104). En este sentido, la 
cultura y la identidad latinoamericana se desarrollan en un contexto en el que los discursos de la modernidad desembocan en el problema del desarrollismo, entendido como la comprensión de las teorías del desarrollo social y la idea del progreso, como mito del desarrollo indefinido, que impacta en el ser humano:

Evidentemente el escenario contemporáneo de América Latina tiene un origen multicausal. Lo que se quiere destacar aquí es el problema de la identidad y de la pertenencia a una cultura para el auténtico desarrollo futuro de la persona y de la comunidad. Los proyectos modernizadores de estirpe racional ilustrada hicieron violencia al ethos cultural de América Latina que buscaba lentamente caminos propios bajo premisas diversas. (Celam, 2005, p. 23)

Vemos aquí, entonces, que en el continente encontramos dos lógicas que se contraponen: la racionalidad instrumental propia de la modernidad ilustrada y sus proyectos desarrollistas que se enfrenta a otro tipo de racionalidad basada en el don, en la gratuidad, en el horizonte de la trascendencia y en la opción preferencial por los pobres. Así lo reconoce el Documento de Medellín: “América Latina está evidentemente bajo el signo de la transformación y el desarrollo. Transformación que, además de producirse con una rapidez extraordinaria, llega a tocar y conmover todos los niveles del hombre, desde el económico hasta el religioso" (Celam, 1968, p. 24).

América Latina se encuentra, por tanto, en medio de las promesas del desarrollo que, en teoría, ayudarían a reducir los índices de pobreza del continente, y que, en el fondo, era uno de los principales problemas denunciados por los sectores que propusieron las revoluciones armadas que se fueron expandiendo por toda la región:

Los desarrollistas se ocupan preferentemente de los medios de producción, que según ellos deben ser modificados en calidad y cantidad. Atribuyen gran valor a la tecnificación y al planteamiento de la sociedad. Sostienen que el pueblo marginado debe ser integrado en la sociedad, como productor y consumidor. Ponen más énfasis en el progreso económico que en la promoción social del pueblo, en vista de la participación de todos en las decisiones que interesan al orden económico y político. (Celam, 1968, n. 4)

La lectura del problema económico en la región tiene repercusiones en lo que se puede comprender como el escándalo del subdesarrollo (Luciani, 2018, pp. 121-138), que tiene profundas repercusiones en el sujeto latinoamericano. 
Este, relacionado con la cultura, implica la comprensión del esfuerzo del pueblo latinoamericano de encontrar las fuentes del sentido y del significado para la vida personal y comunitaria de las personas, que sea capaz de integrar las ventajas de los discursos desarrollistas, pero que a su vez implique el crecimiento de la persona y de la comunidad. Así lo propone Rodrigo Guerra: "Significa recuperar el sentido comunitario de la vida personal y eclesial como camino pedagógico permanente" (Celam, 2005, p. 104). Un camino pedagógico que sea lo suficientemente crítico como para dar cuenta de los elementos que se van incorporando a la cultura y al final no aportan al crecimiento de la persona ni de la sociedad; un camino que sea lo suficientemente liberador para apelar a la praxis, a la transformación cultural en las prácticas comunitarias.

\section{La II Conferencia General del Episcopado Latinoamericano y la lectura de la realidad de la región}

La II Conferencia recibe un concilio cuya intención era proponer una pastoral a la altura de los tiempos. Aunque tuvo defensores como detractores, no estaba en cuestión la necesidad o no del Concilio, sino, en el fondo, cómo debía ser juzgado el mundo contemporáneo. Los documentos plantean una nueva relación entre Iglesia-mundo, en la que se debatía lo que era vinculante e inmutable en la Iglesia y lo que en ella estaba ligado al tiempo y era mutable y, por consiguiente, debía ser reformado (Pottmeyer, 2016, p. 639). La conferencia de Medellín recibe un concilio que apenas comienza a interpretarse. Si bien el concepto de hermenéutica de la refor$m a$ fue posterior, utilizado por el papa Benedicto XVI (2005), el trabajo de los obispos en esta conferencia se tornó en cómo interpretar las propuestas del Concilio en el continente latinoamericano. En este sentido, fueron de gran ayuda los avances en las ciencias bíblicas, que aportaron en dilucidar, al menos en parte, qué tanto de divino y qué tanto de humano había en las escrituras para nuestra salvación.

De esta manera, interpretar el mundo hace parte de la hermenéutica de la reforma que ha practicado el Concilio. Quien reflexione hoy sobre la Iglesia no puede dejar de comprender el mundo y la sociedad actual. De ahí que la categoría secularización puede ayudar a realizar este ejercicio hermenéutico sin renunciar a las categorías teológicas. Llama la atención la lectura de la II Conferencia sobre una no muy positiva relación entre la teología y otras ciencias en su momento, que da cuenta de "una insuficiencia en la instauración del diálogo entre la 
Teología y las diversas ramas del saber, que respete la debida autonomía de las ciencias y aporte la luz del Evangelio para la convergencia de los valores humanos en Cristo" (Celam, 1968, n. 7).

Este ejercicio hermenéutico no fue asunto fácil para una iglesia que ahora quería interpretar el mundo. Son bien conocidas las rupturas, en especial de los sectores de la Iglesia de mayor tradición: "voces dotadas de autoridad en la Iglesia ponen en guardia contra una adaptación al mundo actual. Se llegó a una polarización entre los miembros de diferentes iglesias locales, sobre todo en el mundo occidental" (Pottmeyer, 2016, p. 639). Estas tensiones también serían evidentes en las conferencias siguientes a la de Medellín.

En el Vaticano II, se afirma la conciencia del cristianismo en la historia. Más allá de la complejidad de hacer una lectura unificadora de toda una religión hacia finales de la década de 1960, podemos afirmar que "la Iglesia católica ha tratado de un progresivo repliegue ante un mundo complejo, en el que el catolicismo se sentía, en parte, extraño y desplazado" (Riccardi, 2016, p. 618). La idea clásica del mundo y de la Iglesia se había derrumbado, pero las ideas del nuevo mundo y de la misma Iglesia aún están por construirse. Así lo veía Pablo VI en Populorum progressio:

Es verdad que el mundo se encuentra en un lamentable vacío de ideas. Nos hacemos un llamamiento a los pensadores de Dios, ávidos de absoluto, de justicia y de verdad: todos los hombres de buena voluntad. A ejemplo de Cristo, nos atrevemos a rogaros con insistencia: "buscad y hallareis" (Lc 11,9), emprended los caminos que conducen, a través de la colaboración, de la profundización del saber, de la amplitud del corazón, a una vida más fraterna. (Paulo VI, 1967, n. 85)

A la Iglesia posconciliar le corresponde pensarse en el siglo más secularizado de la historia, hasta el punto de ver realizado el primer Estado integralmente ateo con la Albania de 1967. El Vaticano II plantea el otro (los musulmanes, los ateos, los protestantes) como al que ya no hay que oponerse, como se había planteado, sino como alguien con quien hay que cohabitar pacíficamente. De esta manera, el Vaticano II da paso a la libertad religiosa como una nueva forma de comprender la Iglesia en el mundo, cohabitando con las demás religiones. Medellín reconoce la Iglesia en y para el mundo, y no fuera, al centro o en contra de él (Legoreta, 2014, pp. 653-666).

Sin embargo, el Vaticano II, al intentar el diálogo interreligioso o ecumenismo, no había previsto la diversidad de escenarios y mutaciones religiosas: el movimiento neoprotestante, la teología de la prosperidad, las religiones marcadas por el fundamentalismo y la estrecha relación contemporánea entre religión 
y política. En la época del Concilio, la previsión que se hacía era la autoafirmación de la Iglesia de un mundo secular, no la de un mundo pluralmente religioso o de lenguajes religiosos, como ha acontecido, sin embargo, en gran parte de los casos. En el caso del protestantismo, este experimenta - por decirlo de una manera genérica - el periodo de mayor crecimiento en el siglo XX: desde una poca feligresía reducida a los países históricamente protestantes a quinientos millones en un siglo (Riccardi, 2016, p. 618). Esto plantea grandes retos para la pastoral latinoamericana, y de ahí que pueda ser útil la comprensión de la categoría de la secularización, para entender las transformaciones del mapa religioso contemporáneo. De hecho, Medellín es, a mi parecer, profética, porque logra plantear la evangelización más allá del binomio creencia-increencia:

La actitud de la Iglesia en el campo de la educación, no puede ser la de contraponer la escuela confesional a la no confesional, la escuela - privada - a la oficial, sino la de colaboración abierta y franca entre escuela y escuela, universidad y universidad, entre las escuelas y las iniciativas extraescolares de formación de educación, entre los planes de educación de la Iglesia y los del Estado; "colaboración que exige el bien de la comunidad universal de los hombres". Esta coordinación no constituye peligro para el carácter confesional de las escuelas católicas; antes bien es un deber post-conciliar de las mismas, según el nuevo concepto de presencia de la Iglesia en el mundo de hoy. (Celam, 1968, n. 29)

\section{La secularización como lectura sociológica del problema entre religión y modernidad}

La secularización nos coloca históricamente en los cambios que se evidencian desde el proceso de globalización si hacemos un acercamiento más contemporáneo o podemos rastrear las raíces de estos desde el comienzo de la Ilustración. Las aproximaciones para interpretar esos nuevos cambios fueron incipientes desde las primeras conferencias, pero en la dirección correcta:

Si bien en el siglo $X X$ la realización de las conferencias episcopales había ofrecido un mapeo de sujetos católicos, carecemos aún de la reflexión sobre la globalización, realidad en la que ambientar la Iglesia y la comunión entre iglesias [...] La geografía de lo humano y del habitar ha cambiado profundamente. (Riccardi, 2016, p. 608) 
En este sentido, ampliar la mirada teológica a través de la categoría de la secularización puede dar pistas de la realidad religiosa del continente.

Desde de la Ilustración, la teoría de la secularización consideraba que, a medida que avanzaba la modernidad, la humanidad cada vez más se distanciaría de las estructuras religiosas. El hombre moderno sería aquel que podría vivir sin las preguntas por lo absoluto. Sin embargo, una rápida lectura de la realidad da cuenta de que vivimos en un mundo todavía religioso. Frente a este "retorno" de lo religioso, las teorías de la secularización tuvieron que ser revisadas. Peter Berger afirma que la tesis de la secularización según la cual la religión retrocede a medida que avanza la modernidad es falsa, y que el mundo actual, excepto casos contados, es un mundo netamente religioso. Esta declaración le implicó retractarse de gran parte de su propia producción teórica sobre el tema (Beltrán, 2007). Para Berger y Luckman (1997), la permanencia de lo religioso en las sociedades se explica en la medida en la que las instituciones religiosas son proveedoras de sentido tanto a nivel existencial del individuo como en sentido general de la comunidad.

En el contexto francés, Ferry (1999) ha llamado a este movimiento salida de la religión y permanencia de lo religioso. Todo depende de lo que se entienda por sagrado, divino, religioso, espiritual. En esta reflexión, se encuentra un proceso de humanización de lo divino y divinización de lo humano. De esta manera, se puede descubrir lo religioso a partir de las experiencias completamente autónomas (Gauchet y Ferry, 2007), sin que necesariamente pertenezcan al pasado o asociado a la institución religiosa. Según estas experiencias, lo religioso adquiere dimensión de presente o de futuro. Para Gauchet y Ferry, asistimos a un doble proceso:

A una salida de la religión, entendida como la salida de la capacidad de lo religioso para estructurar la política y la sociedad, y a una permanencia de lo religioso, en el orden de la convicción última de los individuos [...] esto es, la preocupación por las cuestiones últimas, las cuestiones que tratan del destino humano, del significado de las experiencias fundamentales de la vida humana y de la orientación de la ética global de la existencia. (p. 120)

Otra visión de la secularización es la que ha realizado Taylor (2015), quien ha dedicado sus últimas publicaciones a estudiar el hecho religioso. Para Taylor, la secularización es un proceso innovador de la transformación de las condiciones sociales e individuales de la experiencia religiosa, que no es resultado de una racionalidad absoluta, y que se sitúa en una experiencia plurívoca, siempre abierta a su interpretación. Normalmente se sitúa la secularización desde el acontecimiento de la Ilustración del siglo XVI. Sin embargo, Taylor advierte que no es 
posible entender la secularización como una transición simple y lineal de la trascendencia a la increencia o de la religión a la irreligión. Se comprende entonces la secularización como el desarrollo de un ambiente espiritual muy articulado, en el que las alternativas se multiplican; y todas ellas a su modo ejercen una seducción en los individuos. Esta diversidad tiene que ver de igual manera con las variadas formas posibles de comprender la modernidad y la laicidad, es decir, no es posible un único modelo de laicidad.

Taylor (2015) se propone ver el orden secular como el arribo de un nuevo horizonte moral: el humanismo exclusivo. Así, el contexto secular no consiste simplemente en la ausencia de fe o en la simple indiferencia, sino en una condición histórica con profundas motivaciones epistemológicas, pero también morales y espirituales que condicionan tanto al creyente como al no creyente. De esta manera, Taylor encara el problema del secularismo desde una nueva perspectiva, no como un fin en sí mismo, tampoco en función de la restricción de las religiones. Más bien, el secularismo está al servicio de la realización de los plurales bienes morales de la modernidad, todos ellos profundamente conflictivos y dilemáticos (Niño, 2012).

De hecho, lo secular está asociado a una categoría cristiana, en el que el islam parece no disponer de una categoría que cuente con la noción de diferencia entre la Iglesia y el Estado. Esto significaría que las sociedades musulmanas no podrían adoptar regímenes seculares o laicos. Aquí es donde el concepto como tal comienza a ser problemático; si bien la palabra sigue usándose, los fenómenos que intentaban explicar han venido cambiando. De hecho, hay quienes proponen que se hable ya no se secularización, sino de una secularización conflictiva (De la Cueva, 2015), segunda secularización (Marramao y Arroyo, 2017) o postsecularización (Garzón, 2014).

En relación con la categoría signos de los tiempos, aunque su origen es bíblico, su desarrollo aparecerá posteriormente en el magisterio de Juan XXIII y Paulo VI. La adopción oficial de la categoría aparece en la constitución pastoral Gaudium et spes, que plantea los ejes Iglesia y mundo en diálogo, antropológicocristológico y, finalmente, el eje histórico-escatológico. Sin embargo, no fue una categoría de fácil adopción por el Concilio, debido al enfoque de su uso en dos planos diferentes: el primero comprendido desde un horizonte bíblico y escatológico, mientras que otros le dieron un uso más pastoral y sociológico.

En tiempos del Concilio, la categoría estuvo impregnada por un excesivo positivismo, contrastado entonces con la rapidez y vertiginosidad de los cambios sociales. Esto implicaba por consiguiente mucha más atención e interpretación 
más allá de los acontecimientos del mundo en sí mismos (Sepúlveda, 2015). En el campo teológico, lo importante es asumir que la clave hermenéutica para comprender no solo la categoría sino la revelación en sí misma es la persona de Jesucristo como signo de los tiempos. La historia de la Iglesia y de la humanidad se lee a partir de la experiencia de la Encarnación y la experiencia pascual. Es desde esta clave de comprensión que se entiende todo el proyecto de Reino de Dios y el proyecto salvador del Padre.

\section{A manera de conclusión}

La II Conferencia del Episcopado Latinoamericano realizada en Medellín dio elementos valiosos para comprender la identidad y la misión de la Iglesia y el continente. Hoy, cincuenta años después, vale la pena reconocer en ella su espíritu profético, su opción por una Iglesia pobre al servicio de los pobres. Ha dado una recepción teológica creativa, original, selectiva, inacabada del Vaticano II, tal como lo pidieron los padres conciliares. Se destaca que todo proceso eclesial, social y educativo debe partir del escenario cultural de los pueblos, entendido como la forma en que se relacionan los individuos en una sociedad completa. Se evidencia la insuficiencia de los programas desarrollistas que no tuvieron en cuenta la cultura como escenario integral de salvación y liberación humana.

El Documento de Medellín logra interpretar el espíritu de la reforma conciliar. Bajo la máxima Ecclesia semper reformanda, la II Conferencia asume la conciencia de una iglesia que propone un camino de renovación. Ya habrá que ver si las conferencias posteriores lograron captar de igual manera el espíritu de la reforma, no solo a través de los documentos, sino a través de todo el trabajo pastoral en el continente. En ese sentido, aún queda la tarea de seguir discerniendo qué es lo reformable en la Iglesia y qué es lo inmutable.

La secularización puede convertirse en una categoría importante para la teología y las ciencias sociales con el fin de comprender las transformaciones sociales que el continente vive en un siglo caracterizado por las dinámicas de transformación de lo religioso. Aparece la necesidad de comprender de forma más amplia, no solo como el simple tránsito de lo creyente a lo no creyente, sino la complejidad de las transformaciones de lo religioso, que requiere una mirada hermenéutica capaz de dar cuenta de estos cambios.

La comprensión de esta categoría puede entrar en diálogo con los criterios hermenéuticos para discernir los signos de los tiempos latinoamericanos (pluralidad regulada de interpretaciones: ni un sentido único, ni infinitos sentidos; el 
sentido de una realidad que tiene otros sentidos, la dialéctica entre acontecimiento y sentido) citados en Azcuy, Schickendantz y Silva (2013). Si bien analizar esta relación excede lo que es posible plantear, quedan formuladas en el futuro para continuar el análisis, particularmente en la relación entre sus métodos: el método sociológico y fenomenológico de la secularización, y por su parte, el método de ver-juzgar-actuar de la categoría teológica de signos de los tiempos.

\section{Referencias}

Azcuy, V. R., Schickendantz, C. y Silva, E. (2013). La teología de los signos de los tiempos: horizontes, criterios y métodos. Santiago de Chile, Chile: Universidad Alberto Hurtado. DOI: https://doi.org/10.1177/0040563914548659h

Beltrán Cely, W. M. (2007). La sociología de la religión: una revisión al estado del arte. En AA. VV., Creer y poder hoy: memorias de la Cátedra Manuel Ancízar (pp. 75-94). Bogotá, Colombia: Universidad Nacional de Colombia.

Benedicto XVI. (2005). Discorso di sua santità Benedetto XVI alla curia romana in occasione della presentazione degli auguri natalizi. Ciudad del Vaticano: Editrice Vaticana.

Berguer, P. L. y Luckman, T. (1997). Modernidad, pluralismo y crisis de sentido. Barcelona, España: Paidós.

Consejo Episcopal Latinoamericano. (1968). Documento conclusivo de Medellín. Bogotá, Colombia: Autor.

Consejo Episcopal Latinoamericano. (2005). La cultura latinoamericana en proceso de transformación. En América Latina: sociedades en cambio (pp. 7-23). Bogotá, Colombia: Autor.

Cueva Merino, J. de la (2015). Conflictiva secularización: sobre sociología, religión e historia. Historia Contemporánea, 51, 365-395. DOI: http://dx.doi.org/10.1387/ hc. 14712

Ferry, L. (1999). El hombre-Dios o el sentido de la vida. Barcelona, España: Paidós.

Garzón Vallejo, I. (2014). Postsecularidad: ¿un nuevo paradigma para las ciencias sociales? Revista de Estudios Sociales, 50, 101-112. DOI: http://dx.doi.org/10.7440/ res50.2014.11

Gauchet, M. y Ferry, L. (2007). Lo religioso después de la religión. Barcelona, España: Antropos.

Habermas, J. (2006). Entre naturalismo y religión. Barcelona, España: Paidós.

Legoreta Zepeda, J. de J. (2014). La Reforma de la Iglesia en los documentos de las conferencias del episcopado latinoamericano. Revista Eclesiástica Brasilera, 295, 653-666. 
Luciani, R. (2018). Medellín 50 años después: del desarrollo a la liberación (I). Teología, 55(125), 121-138.

Marramao, G. y Arroyo, F. (2017). Diálogos: Marramao y Arroyo. Barcelona, España: Gedisa.

Niño Castro, A. (2012). Hacia una nueva comprensión de la modernidad: repensar el secularismo desde A Secular Age de Charles Taylor (Tesis de maestría) Pontificia Universidad Javeriana: Bogotá, Colombia.

Paulo VI. (1967). Populorum progresio. Ciudad del Vaticano: Editrice Vaticana.

Pottmeyer, H. (2016). La Iglesia como camino para configurarse como pueblo de Dios. En A. Spadaro y C. M. Galli (Eds.), La reforma y las reformas en la Iglesia (pp. 79-93). Madrid, España: Sal Terrae.

Riccardi, A. (2016). Lecciones de las reformas del siglo XX. En A. Spadaro y C. M. Galli (Eds.), La reforma y las reformas en la Iglesia (pp. 121-137). Madrid, España: Sal Terrae.

Scatena, S. (2016). De Medellín a Aparecida: la "lección" de una experiencia regional para una búsqueda de formas y estilos de colegialidad efectiva. En A. Spadaro y C. M. Galli (Eds.), La reforma y las reformas en la Iglesia (pp. 273-295). Madrid, España: Sal Terrae.

Sendoya Mejía, L. M. (2011). Fundamentos sociológicos de la ERE. En J. L. Meza Rueda, Educación religiosa escolar (pp. 126-151). Bogotá, Colombia: San Pablo.

Sepúlveda Romero, L. V. (2015). Reseña: La categoría teológica signos de los tiempos: desde el Concilio Vaticano II al Pentecostés de Aparecida y Francisco. Cuestiones Teológicas, 42(98), 572-575. DOI: https://doi.org/10.2307/j.ctvb938v0.7

Taylor, C. (2015). Incanto e disincanto. Bolonia, Italia: Centro Editoriale Dehoniano. 